Research Article

\title{
Sequential Appointment Scheduling Considering Walk-In Patients
}

\author{
Chongjun Yan, ${ }^{1}$ Jiafu Tang, $^{1,2}$ and Bowen Jiang ${ }^{1}$ \\ ${ }^{1}$ Department of Systems Engineering, State Key Lab of Synthetic Automation of Process Industry, Northeastern University (NEU), \\ Shenyang 110004, China \\ ${ }^{2}$ College of Management Science and Engineering, Dongbei University of Finance and Economics, Dalian 116025, China
}

Correspondence should be addressed to Jiafu Tang; jftang@mail.neu.edu.cn

Received 26 September 2013; Accepted 26 February 2014; Published 6 April 2014

Academic Editor: Baocang Ding

Copyright (C) 2014 Chongjun Yan et al. This is an open access article distributed under the Creative Commons Attribution License, which permits unrestricted use, distribution, and reproduction in any medium, provided the original work is properly cited.

\begin{abstract}
This paper develops a sequential appointment algorithm considering walk-in patients. In practice, the scheduler assigns an appointment time for each call-in patient before the call ends, and the appointment time cannot be changed once it is set. Each patient has a certain probability of being a no-show patient on the day of appointment. The objective is to determine the optimal booking number of patients and the optimal scheduling time for each patient to maximize the revenue of all the arriving patients minus the expenses of waiting time and overtime. Based on the assumption that the service time is exponentially distributed, this paper proves that the objective function is convex. A sufficient condition under which the profit function is unimodal is provided. The numerical results indicate that the proposed algorithm outperforms all the commonly used heuristics, lowering the instances of no-shows, and walk-in patients can improve the service efficiency and bring more profits to the clinic. It is also noted that the potential appointment is an effective alternative to mitigate no-show phenomenon.
\end{abstract}

\section{Introduction}

It is routine practice for a clinic patient to make an appointment before seeing a doctor. An effective appointment system provides a balance between revenue and cost in terms of the patients' waiting and clinic overtime. Appointment scheduling problems have received the attention of operations research (OR) scientists since Bailey's initial work in 1952 [1], but the problem of long waiting times still exists in the outpatient clinic. The no-show behavior of patients has been identified as a key factor resulting in low efficiency in many field study papers. Attempts to cut down on no-shows by reminders, telephones, or emails play an important role in mitigating no-show phenomenon, but it cannot eliminate the negative effects entirely. A revolutionary appointment system named the open-access system has been introduced as an alternative method to reduce no-show behavior by giving some patients appointments on the day they call.

Cayirli and Veral [2] provide a comprehensive review of previous studies and classify the environmental factors influencing the problem formulation, commonly used performance measures, the appointment rules, and the research methodologies. Gupta and Denton [3] provide stateof-the-art, new challenges and opportunities for primary care appointment scheduling, specialty clinic appointment scheduling, and elective surgery appointment scheduling. The previous studies can be viewed in three categories. The first category is the appointment capacity planning level, which determines the optimal number of patients to be seen to maximize the total utility or revenue (Kim and Giachetti [4]; Qu et al. [5]; LaGanga and Lawrence [6]); the second category is the appointment scheduling level, which decides the optimal appointment time for each patient or the optimal interval length for a predetermined number of patients in a session (Kaandorp and Koole [7]; Hassin and Mendel [8]; Cayirli et al. [9]); the last category includes the joint decisions of capacity planning and scheduling to search for the optimal number and the corresponding schedule at the same time. (Muthuraman and Lawley [10]; Chakraborty et al. [11, 12]; Zeng et al. [13]; Turkcan et al. [14]). The sequential scheduling technique, which belongs to the last category, is developed in 
recent years to address overbooking and general stochastic service time.

The most relevant study to sequential appointment scheduling is as follows. Muthuraman and Lawley [10] propose a sequential scheduling approach with exponential service times (Kopach et al. [15] displayed the validity of this assumption) to maximize the revenue minus the cost of overflow and tardiness and prove that the objective function is unimodal. Chakraborty et al. [11] generalized Muthuraman and Lawley's [10] model for general distributed service time and proved the unimodality of the profit function as well. Each accepted booking request is arranged in one of the predefined intervals in both papers, which is not required here. Gupta and Wang [16] model appointment sequence considering patients' choice by a Markov decision process. The optimum threshold policies, after which future booking requests are rejected, are derived for single and multiple physicians under the assumption of deterministic service time; this paper includes exponential service time and walkin patients. Erdogan and Denton [17] formulate a two-stage stochastic linear program model that minimizes the expected cost of waiting time, idleness, and overtime considering no-shows for a given appointment sequence. The optimal solution is obtained by a standard L-shaped algorithm and the upper bounds are derived without calculating the revenue by seeing patients. Zeng et al. [13] demonstrate that the profit function of their sequential appointment scheduling problem for homogenous patients is multimodular, but the multimodularity is not preserved under scenarios of heterogeneous patients, and a local search heuristic is developed to find the local optimal schedules. Turkcan et al. [14] build a stochastic multiobjective model to account for the impact of the fairness measure on profit and presented a series of sequential search algorithms to find the Pareto-optimal schedules. Different from Muthuraman and Lawley's [10] model, Chakraborty et al. [12] design a sequential clinical scheduling method without predefined intervals, providing the patient with an exact appointment time before the call ends. The convexity of the total cost and unimodality of the net profit are derived under the assumption of exponentially distributed service time. Walk-in patients are included in none of the above research. As pointed by Cayirli and Veral [2], walk-in patients are prevalent for general practitioners who are responsible for the patients' total care. It is also a common phenomenon for the large hospital in China.

All the previous studies use overbooking to reduce the negative effect of no-shows without incorporating walkin patients. Based on previous work, this paper formulates a stochastic overbooking model for an open-access clinic, which allows for moderate walk-in probability on the appointed day. The numerical results demonstrate the correlation between the total profit and the walk-in, no-show probability. Anticipating future arrivals can be viewed as an alternative method to reduce no-show. The paper is structured as follows. Section 2 presents the basic assumptions and the model formulation considering walk-in patients. Section 3 establishes the theoretical proof that the cost function is convex under the assumption of exponential service time together with a sufficient condition under which

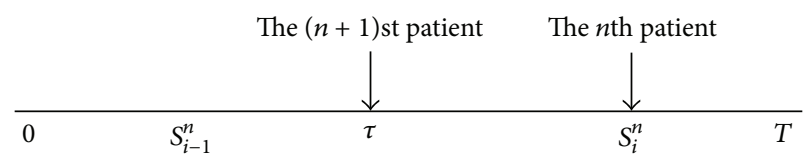

FIGURE 1: Illustration of the sequential appointment problem.

the objective evolution is unimodal. Section 4 provides an illustrative example and parameter impact analysis. Section 5 concludes the main finding and provides future directions.

\section{Assumptions and Model for the Sequential Appointment Problem with Walk-In Patients}

2.1. Assumptions and Parameters. This paper develops a model for a sequential appointment scheduling problem in an open-access clinic, deciding an appointment time for each booking request and a stopping criterion after which the clinic should stop accepting any requests. To provide consistent diagnoses to the patients, every doctor has their own waiting list in realistic situations. A single-server queueing system is utilized to describe the appointment system. Some clinics allow the doctors to conclude the session after the examination of the final patient, but open-access clinics require the doctor to remain available throughout the day to address walk-in patients. It is assumed that the service time is exponentially distributed, and the mean is standardized at 1 . Let $T$ stand for the length of the session, and each booking request will be given an appointment time in $[0, T]$ or rejected. $r$ represents the revenue from an individual patient, and the cost of waiting and overtime per unit of time are denoted by $c_{w}$ and $c_{o}$, respectively. $t_{n}$ stands for the optimal appointment time assigned to the $n$th call-in patient, and $S^{n}$ is the optimal schedule after $n$ patients are arranged, where $S_{i}^{n}$ is the appointment time for the $n$th scheduled patient, $S_{n+1}^{n}=T$, and $S_{i}^{n} \leq S_{i+1}^{n}$. The $n$th scheduled patient is not identical to the $n$th call-in patient, as illustrated in Figure 1 , which describes the scenario that the $n$th call-in patient is the $i$ th scheduled patient and the $n+1$ st patient is attempted to be arranged in $\tau \in\left[S_{i-1}^{n}, S_{i}^{n}\right]$. This updated schedule is denoted by $\Pi_{i-1}^{n+1}$. It is assumed that the patients can be categorized into $J$ groups according to no-show probabilities, which can be inferred using statistics. Let $\theta_{j}^{n}$ be the showing probability of the $n$th call-in patient if he or she is of type $j$ and let $\hat{\theta}_{i}^{n}$ be the showing probability at $S_{i}^{n}$. $\eta$ gives the walkin probability, and it is assumed that $\eta \leq 0.5$. Cayirli et al. [9] propose a procedure to calculate the walk-in probability based on historical data. The probability, represented by $p_{i k}^{n}$, that $k$ patients actually arrive at $S_{i}^{n}$ for the appointment can be determined from formula (1). The first line represents the probability of no arrivals, which is the joint probability of no-show and no walk-in appointments. According to the assumption, it is easy to obtain $p_{i 1}^{n} \geq p_{i 2}^{n}$ from formula (1). This is reasonable because too many walk-ins bring 
large uncertainty in the daily workload, which is the main deficiency of open-access appointment system. Consider

$$
p_{i k}^{n}= \begin{cases}\left(1-\widehat{\theta}_{i}^{n}\right)(1-\eta), & k=0, \\ \left(1-\widehat{\theta}_{i}^{n}\right) \eta+\widehat{\theta}_{i}^{n}(1-\eta), & k=1, \\ \widehat{\theta}_{i}^{n} \eta, & k=2 .\end{cases}
$$

Let $X_{i}^{n}$ denote the number of patients in the system at $S_{i}^{n}$ before new arrivals; obviously, $X_{i}^{n} \leq 2 i-2$; then $Q_{i h}^{n}=$ $\operatorname{Pr}\left\{X_{i}^{n}=h\right\} . Y(t)$ is a random variable subject to Poisson distribution, representing the number of patients seen during time $t$. Define $f_{i l}^{n}=\operatorname{Pr}\left\{Y\left(S_{i}^{n}-S_{i-1}^{n}\right)=l\right\}$ and $F_{i l}^{n}=\operatorname{Pr}\left\{Y\left(S_{i}^{n}-\right.\right.$ $\left.\left.S_{i-1}^{n}\right) \geq l\right\}$. Consider

$$
A_{m}^{n}= \begin{cases}A_{2 n-2}^{n-1} p_{\xi 2}^{n}, & m=2 n, \\ A_{2 n-2}^{n-1} p_{\xi 1}^{n}+A_{2 n-3}^{n-1} p_{\xi 2}^{n}, & m=2 n-1, \\ A_{m}^{n-1} p_{\xi 0}^{n}+A_{m-1}^{n-1} p_{\xi 1}^{n}+A_{m-2}^{n-1} p_{\xi 2}^{n}, & \text { otherwise } \\ A_{1}^{n-1} p_{\xi 0}^{n}+A_{0}^{n-1} p_{\xi 1}^{n}, & m=1, \\ A_{0}^{n-1} p_{\xi 0}^{n}, & m=0 .\end{cases}
$$

$R^{n}$ is the total expected arrivals after $n$ patients are scheduled; then $A_{m}^{n}=\operatorname{Pr}\left\{R^{n}=m\right\}$. If $t_{n}=S_{\xi}^{n}$, that is, the $n$th callin patient is the $\xi$ th scheduled patient, $A_{m}^{n}$ can be calculated recursively based on $A_{m}^{n-1}$ as follows.

$O^{n}$ denotes the overtime after $n$ call-ins, $W_{i}^{n}$ denotes the waiting time at $S_{i}^{n}$, and $P\left(S^{n}\right)$ represents the total net profit of schedule $S^{n}$, with $C\left(S^{n}\right)$ denoting the total cost.

2.2. Model. The key point of modeling sequential appointment scheduling problem is the transition matrix from $X_{i-1}^{n}$ to $X_{i}^{n}$. With the initial condition that $Q_{10}^{n}=1$, another $Q_{i h}^{n}$ can be iteratively derived from $Q_{(i-1) h}^{n}$ as follows.

Formula (3) means that when $h \geq 2$, the probability of $h$ patients in the waiting list (including the one in service) at $S_{i}^{n}$ before new arrivals comprises three terms: the first term is the case that $\kappa$ patients remain waiting at $S_{i-1}^{n}$ before new arrivals, no new patients come at $S_{i-1}^{n}$, and $\kappa-h$ services are completed during $S_{i}^{n}-S_{i-1}^{n}$; subsequent term represents the joint probability that there are $\kappa$ patients at $S_{i-1}^{n}, 1$ new patient arrives at $S_{i-1}^{n}$, and $\kappa-h+1$ services are completed during $S_{i}^{n}-S_{i-1}^{n}$; likewise, the last term has the same implication except that there are 2 new arrivals at $S_{i-1}^{n}$ and $\kappa-h+2$ service completion during $S_{i}^{n}-S_{i-1}^{n}$. By the same means, one can obtain the probability when $j=0,1$. Consider

$$
\begin{aligned}
Q_{i h}^{n}= & \sum_{\kappa=h}^{2 i-4} Q_{(i-1) \kappa}^{n} p_{(i-1) 0}^{n} f_{i(\kappa-h)}^{n}+\sum_{\kappa=h-1}^{2 i-4} Q_{(i-1) \kappa}^{n} p_{(i-1) 1}^{n} f_{i(\kappa+1-h)}^{n} \\
& +\sum_{\kappa=h-2}^{2 i-4} Q_{(i-1) \kappa}^{n} p_{(i-1) 2}^{n} f_{i(\kappa+2-h)}^{n}, \quad h \geq 2, Q_{i 1}^{n} \\
= & \sum_{\kappa=1}^{2 i-4} Q_{(i-1) \kappa}^{n} p_{(i-1) 0}^{n} f_{i(\kappa-1)}^{n}+\sum_{\kappa=0}^{2 i-4} Q_{(i-1) \kappa}^{n} p_{(i-1) 1}^{n} f_{i \kappa}^{n} \\
& +\sum_{\kappa=0}^{2 i-4} Q_{(i-1) \kappa}^{n} p_{(i-1) 2}^{n} f_{i(\kappa+1)}^{n}, Q_{i 0}^{n}
\end{aligned}
$$

$$
\begin{aligned}
= & \sum_{\kappa=0}^{2 i-4} Q_{(i-1) \kappa}^{n} P_{(i-1) 0}^{n} F_{i \kappa}^{n}+\sum_{\kappa=0}^{2 i-4} Q_{(i-1) \kappa}^{n} p_{(i-1) 1}^{n} F_{i(\kappa+1)}^{n} \\
& +\sum_{\kappa=0}^{2 i-4} Q_{(i-1) \kappa}^{n} p_{(i-1) 2}^{n} F_{i(\kappa+2)}^{n} .
\end{aligned}
$$

The expected waiting time at $S_{i}^{n}$ after $n$ call-ins is calculated by

$$
\begin{aligned}
E\left(W_{i}^{n}\right) & =p_{i 1}^{n} E\left(X_{i}^{n}\right)+p_{i 2}^{n}\left(2 E\left(X_{i}^{n}\right)+1\right) \\
& =\left(p_{i 1}^{n}+2 p_{i 2}^{n}\right) \sum_{h=1}^{2 i-2} h Q_{i h}^{n}+p_{i 2}^{n} .
\end{aligned}
$$

Similarly, the expected overtime after $n$ patients are scheduled is given by

$$
E\left(O^{n}\right)=E\left(X_{n+1}^{n}\right)=\sum_{h=1}^{2 n} h Q_{(n+1) h}^{n}
$$

The sequential appointment scheduling problem is to determine the optimal number of booking requests and how to schedule these patients in a session day towards maximizing the total net profit, which is the difference between the revenue and total cost:

$$
\max P\left(S^{n}\right)=r E\left(R^{n}\right)-c_{w} \sum_{i=1}^{n} E\left(W_{i}^{n}\right)-c_{o} E\left(O^{n}\right)
$$

where $E\left(R^{n}\right)=\sum_{m=1}^{2 n} m A_{m}^{n}$.

Of course, when $n$ is fixed, the objective function is equivalent to minimizing the total cost as follows:

$$
\min C\left(S^{n}\right)=c_{w} \sum_{i=1}^{n} E\left(W_{i}^{n}\right)+c_{o} E\left(O^{n}\right)
$$

\section{Sequential Appointment Scheduling Algorithm}

\subsection{Characteristics of the Objective Function}

Proposition 1. The first patient is scheduled at time 0 .

Proof. Suppose that the first patient is scheduled at time $\tau$; then formula (3) can be simplified as

$$
\begin{gathered}
Q_{10}^{1}=1, \\
Q_{T 1}^{1}=p_{11}^{1} \operatorname{Pr}\{Y(T-\tau)=0\}+p_{12}^{1} \operatorname{Pr}\{Y(T-\tau)=1\}, \\
Q_{T 2}^{1}=p_{12}^{1} \operatorname{Pr}\{Y(T-\tau)=0\} .
\end{gathered}
$$


From (4), (5), and (8), the waiting time and overtime can be calculated as

$$
\begin{gathered}
E\left(W_{1}^{1}\right)=p_{12}^{1} \\
E\left(O^{1}\right)=p_{11}^{1} e^{-(T-\tau)}+p_{12}^{1}(T-\tau) e^{-(T-\tau)}+2 p_{12}^{1} e^{-(T-\tau)} .
\end{gathered}
$$

Differentiating the total cost so far with respect to $\tau$, $c_{o}\left(p_{11}^{1} e^{-(T-\tau)}+p_{12}^{1}(T-\tau) e^{-(T-\tau)}+p_{12}^{1} e^{-(T-\tau)}\right)>0$. Then the total cost is monotonically increasing with respect to $\tau$, and, to minimize the total cost, the first patient is scheduled to time $0, t_{1}=0$.

Next, it is proved that the total cost is convex function for $\tau \in\left[S_{i-1}^{n}, S_{i}^{n}\right]$. Central to the proof are the first-order and the second-order derivatives of $W_{i}^{n}$ and $O^{n}$. Firstly, it is shown that the waiting time at time $\tau$ is decreasing convex function of $\tau$. Secondly, it is noted that the waiting time at time $S_{i}^{n}$ is increasing convex function of $\tau$. Thirdly, it is proved that the waiting time at time $S_{c}^{n}(\zeta>i)$ is increasing convex function of $\tau$ by induction. Finally, the overtime is demonstrated to be an increasing convex function of $\tau$.

Theorem 2. For schedule $S^{n}$, the cost function is convex of $\tau$, if the $n+1$ st patient is arranged at $\tau \in\left[S_{i-1}^{n}, S_{i}^{n}\right]$.

Proof. It is easy to get that $\Pi_{i-1}^{n+1}=\left\{\pi_{1}^{n+1}, \pi_{2}^{n+1}, \ldots, \pi_{n+2}^{n+1}\right\}$ satisfies $\pi_{\varsigma}^{n+1}=S_{\zeta}^{n}, \zeta \leq i-1 ; \pi_{i}^{n+1}=\tau ; \pi_{l}^{n+1}=S_{\zeta-1}^{n}, \zeta \geq i+1$. The system parameters corresponding to $\Pi_{i-1}^{n+1}$ are $\widetilde{p}_{\zeta k}^{n+1}, \widetilde{X}_{\varsigma}^{n+1}$, $\widetilde{Q}_{\zeta h}^{n+1}, \widetilde{f}_{\zeta l}^{n+1}, \widetilde{F}_{\zeta l}^{n+1}, \widetilde{W}_{\zeta}^{n+1}$, and $\widetilde{O}^{n+1}$, for $\zeta \in[1, n+1]$. Because the system states prior to $\tau$ are not influenced by the new arrival, the cost will not change. The cost function since $\tau$ $\left(\pi_{\varsigma}^{n+1}, \zeta \geq i\right)$ is classified to four parts.

(1) The waiting time at time $\tau\left(\pi_{\varsigma}^{n+1}, \zeta=i\right)$ is monotonically decreasing convex function of $\tau$.

For $h \geq 2$, from formula (3),

$$
\begin{aligned}
\frac{d}{d \tau} \widetilde{Q}_{i h}^{n+1}= & \sum_{\kappa=h+1}^{2 i-4} \widetilde{Q}_{(i-1) \kappa}^{n+1} \widetilde{p}_{(i-1) 0}^{n+1}\left(\widetilde{f}_{i(\kappa-h-1)}^{n+1}-\widetilde{f}_{i(\kappa-h)}^{n+1}\right) \\
& -\widetilde{Q}_{(i-1) h}^{n+1} \widetilde{p}_{(i-1) 0}^{n+1} \widetilde{f}_{i 0}^{n+1} \\
& +\sum_{\kappa=h}^{2 i-4} \widetilde{Q}_{(i-1) \kappa}^{n+1} \widetilde{p}_{(i-1) 1}^{n+1}\left(\widetilde{f}_{i(\kappa-h)}^{n+1}-\widetilde{f}_{i(\kappa-h+1)}^{n+1}\right) \\
& \quad-\widetilde{Q}_{(i-1)(h-1)}^{n+1} \widetilde{p}_{(i-1) 1}^{n+1} \widetilde{f}_{i 0}^{n+1} \\
& \sum_{\kappa=h-1}^{2 i-4} \widetilde{Q}_{(i-1) \kappa}^{n+1} \widetilde{p}_{(i-1) 2}^{n+1}\left(\widetilde{f}_{i(\kappa-h+1)}^{n+1}-\widetilde{f}_{i(\kappa-h+2)}^{n+1}\right) \\
& -\widetilde{Q}_{(i-1)(h-2)}^{n+1} \widetilde{p}_{(i-1) 2}^{n+1} \widetilde{f}_{i 0}^{n+1}=\widetilde{Q}_{i(h+1)}^{n+1}-\widetilde{Q}_{i h}^{n+1} .
\end{aligned}
$$

Likewise,

$$
\begin{array}{r}
\frac{d}{d \tau} \widetilde{Q}_{i 1}^{n+1}=\sum_{\kappa=2}^{2 i-4} \widetilde{Q}_{(i-1) \kappa}^{n+1} \widetilde{p}_{(i-1) 0}^{n+1}\left(\widetilde{f}_{i(\kappa-2)}^{n+1}-\widetilde{f}_{i(\kappa-1)}^{n+1}\right) \\
\quad-\widetilde{Q}_{(i-1) 1}^{n+1} \widetilde{p}_{(i-1) 0}^{n+1} \widetilde{f}_{i 0}^{n+1} \\
\quad+\sum_{\kappa=1}^{2 i-4} \widetilde{Q}_{(i-1) \kappa}^{n+1} \widetilde{p}_{(i-1) 1}^{n+1}\left(\widetilde{f}_{i(\kappa-1)}^{n+1}-\widetilde{f}_{i \kappa}^{n+1}\right) \\
\quad-\widetilde{Q}_{(i-1) 0}^{n+1} \widetilde{p}_{(i-1) 1}^{n+1} \widetilde{f}_{i 0}^{n+1} \\
\quad+\sum_{\kappa=0}^{2 i-4} \widetilde{Q}_{(i-1) \kappa}^{n+1} \widetilde{p}_{(i-1) 2}^{n+1}\left(\widetilde{f}_{i \kappa}^{n+1}-\widetilde{f}_{i(\kappa+1)}^{n+1}\right) \\
=\widetilde{Q}_{i 2}^{n+1}-\widetilde{Q}_{i 1}^{n+1}, \quad \therefore \sum_{h=0}^{2 i-2} \frac{d}{d \tau} \widetilde{Q}_{i h}^{n+1}=0 . \\
\because \sum_{h=0}^{2 i-2} \widetilde{Q}_{i h}^{n+1}=1, \quad \therefore
\end{array}
$$

From (10), (11), and (12), it is simple to derive

$$
\frac{d}{d \tau} \widetilde{Q}_{i 0}^{n+1}=\widetilde{Q}_{i 1}^{n+1}
$$

Using (10) and (11), the first-order and second-order derivatives of $E\left(\widetilde{X}_{i}^{n+1}\right)$ are calculated by

$$
\begin{aligned}
\frac{d}{d \tau} E\left(\widetilde{X}_{i}^{n+1}\right) & =\sum_{h=1}^{2 i-2} h \frac{d}{d \tau} \widetilde{Q}_{i h}^{n+1}=\sum_{h=1}^{2 i-2} h\left(\widetilde{Q}_{i(h+1)}^{n+1}-\widetilde{Q}_{i h}^{n+1}\right) \\
& =\sum_{h=1}^{2 i-2} h \widetilde{Q}_{i(h+1)}^{n+1}-\sum_{h=1}^{2 i-2} h \widetilde{Q}_{i h}^{n+1}=-\sum_{h=1}^{2 i-2} \widetilde{Q}_{i h}^{n+1}<0 \\
\frac{d^{2}}{d \tau^{2}} E\left(\widetilde{X}_{i}^{n+1}\right) & =-\sum_{h=1}^{2 i-2} \frac{d}{d \tau} \widetilde{Q}_{i h}^{n+1} \\
& =-\sum_{h=1}^{2 i-2}\left(\widetilde{Q}_{i(h+1)}^{n+1}-\widetilde{Q}_{i h}^{n+1}\right)=\widetilde{Q}_{i 1}^{n+1}>0
\end{aligned}
$$

Then, from formula (4), the result is established by

$$
\frac{d}{d \tau} E\left(\widetilde{W}_{i}^{n+1}\right)<0, \quad \frac{d^{2}}{d \tau^{2}} E\left(\widetilde{W}_{i}^{n+1}\right)>0
$$

(2) The waiting time at time $S_{i}^{n}\left(\pi_{c}^{n+1}, \zeta=i+1\right)$ is monotonically increasing convex function of $\tau$. 
For $h \geq 3$, formula (3) is reduced to

$$
\begin{aligned}
\frac{d}{d \tau} \widetilde{Q}_{(i+1) h}^{n+1}= & \sum_{\kappa=h+1}^{2 i-2} \widetilde{Q}_{i k}^{n+1} \widetilde{p}_{i 0}^{n+1}\left(\widetilde{f}_{(i+1)(\kappa-h)}^{n+1}-\widetilde{f}_{(i+1)(\kappa-h-1)}^{n+1}\right) \\
& +\sum_{\kappa=h+1}^{2 i-2}\left(\widetilde{Q}_{i(\kappa+1)}^{n+1}-\widetilde{Q}_{i \kappa}^{n+1}\right) \widetilde{p}_{i 0}^{n+1} \widetilde{f}_{(i+1)(\kappa-h)}^{n+1} \\
& +\widetilde{Q}_{i h}^{n+1} \widetilde{p}_{i 0}^{n+1} \widetilde{f}_{(i+1) 0}^{n+1}+\left(\widetilde{Q}_{i(h+1)}^{n+1}-\widetilde{Q}_{i h}^{n+1}\right) \widetilde{p}_{i 0}^{n+1} \widetilde{f}_{(i+1) 0}^{n+1} \\
& +\sum_{\kappa=h}^{2 i-2} \widetilde{Q}_{i \kappa}^{n+1} \widetilde{p}_{i 1}^{n+1}\left(\widetilde{f}_{(i+1)(\kappa-h+1)}^{n+1}-\widetilde{f}_{(i+1)(\kappa-h)}^{n+1}\right) \\
& +\sum_{\kappa=h}^{2 i-2}\left(\widetilde{Q}_{i(\kappa+1)}^{n+1}-\widetilde{Q}_{i \kappa}^{n+1}\right) \widetilde{p}_{i 1}^{n+1} \widetilde{f}_{(i+1)(\kappa-h+1)}^{n+1} \\
& +\widetilde{Q}_{i(h-1)}^{n+1} \widetilde{p}_{i 1}^{n+1} \widetilde{f}_{(i+1) 0}^{n+1} \\
& +\left(\widetilde{Q}_{i h}^{n+1}-\widetilde{Q}_{i(h-1)}^{n+1}\right) \widetilde{p}_{i 1}^{n+1} \widetilde{f}_{(i+1) 0}^{n+1} \\
& +\sum_{\kappa=h-1}^{2 i-2} \widetilde{Q}_{i \kappa}^{n+1} \widetilde{p}_{i 2}^{n+1}\left(\widetilde{f}_{(i+1)(\kappa-h+2)}^{n+1}-\widetilde{f}_{(i+1)(\kappa-h+1)}^{n+1}\right) \\
& +\sum_{\kappa=h-1}^{2 i-2}\left(\widetilde{Q}_{i(\kappa+1)}^{n+1}-\widetilde{Q}_{i \kappa}^{n+1}\right) \widetilde{p}_{i 2}^{n+1} \widetilde{f}_{(i+1)(\kappa-h+2)}^{n+1} \\
& +\widetilde{Q}_{i(h-2)}^{n+1} \widetilde{p}_{i 2}^{n+1} \widetilde{f}_{(i+1) 0}^{n+1} \\
& \left(\widetilde{Q}_{i(h-1)}^{n+1}-\widetilde{Q}_{i(h-2)}^{n+1}\right) \widetilde{p}_{i 2}^{n+1} \widetilde{f}_{(i+1) 0}^{n+1}=0 . \\
& ={ }^{2 i+1}(16)
\end{aligned}
$$

Using formula (3), further result is expressed as

$$
\frac{d}{d \tau} \widetilde{Q}_{(i+\kappa) h}^{n+1}=0, \quad \forall \kappa \geq 1, h \geq 2 \kappa+1 .
$$

Accordingly, when $h=2,1$,

$$
\begin{aligned}
\frac{d}{d \tau} \widetilde{Q}_{(i+1) 2}^{n+1}= & \sum_{\kappa=3}^{2 i-2} \widetilde{Q}_{i \kappa}^{n+1} \widetilde{p}_{i 0}^{n+1}\left(\widetilde{f}_{(i+1)(\kappa-2)}^{n+1}-\widetilde{f}_{(i+1)(\kappa-3)}^{n+1}\right) \\
& +\sum_{\kappa=3}^{2 i-2}\left(\widetilde{Q}_{i(\kappa+1)}^{n+1}-\widetilde{Q}_{i \kappa}^{n+1}\right) \widetilde{p}_{i 0}^{n+1} \widetilde{f}_{(i+1)(\kappa-2)}^{n+1} \\
& +\widetilde{Q}_{i 2}^{n+1} \widetilde{p}_{i 0}^{n+1} \widetilde{f}_{(i+1) 0}^{n+1}+\left(\widetilde{Q}_{i 3}^{n+1}-\widetilde{Q}_{i 2}^{n+1}\right) \widetilde{p}_{i 0}^{n+1} \widetilde{f}_{(i+1) 0}^{n+1} \\
& +\sum_{\kappa=2}^{2 i-2} \widetilde{Q}_{i \kappa}^{n+1} \widetilde{p}_{i 1}^{n+1}\left(\widetilde{f}_{(i+1)(\kappa-1)}^{n+1}-\widetilde{f}_{(i+1)(\kappa-2)}^{n+1}\right) \\
& +\sum_{\kappa=2}^{2 i-2}\left(\widetilde{Q}_{i(\kappa+1)}^{n+1}-\widetilde{Q}_{i \kappa}^{n+1}\right) \widetilde{p}_{i 1}^{n+1} \widetilde{f}_{(i+1)(\kappa-1)}^{n+1} \\
& +\widetilde{Q}_{i 1}^{n+1} \widetilde{p}_{i 1}^{n+1} \widetilde{f}_{(i+1) 0}^{n+1}+\left(\widetilde{Q}_{i 2}^{n+1}-\widetilde{Q}_{i 1}^{n+1}\right) \widetilde{p}_{i 1}^{n+1} \widetilde{f}_{(i+1) 0}^{n+1} \\
& +\sum_{\kappa=1}^{2 i-2} \widetilde{Q}_{i \kappa}^{n+1} \widetilde{p}_{i 2}^{n+1}\left(\widetilde{f}_{(i+1) \kappa}^{n+1}-\widetilde{f}_{(i+1)(\kappa-1)}^{n+1}\right) \\
& +\sum_{\kappa=1}^{2 i-2}\left(\widetilde{Q}_{i(\kappa+1)}^{n+1}-\widetilde{Q}_{i \kappa}^{n+1}\right) \widetilde{p}_{i 2}^{n+1} \widetilde{f}_{(i+1) \kappa}^{n+1} \\
& +\widetilde{Q}_{i 0}^{n+1} \widetilde{p}_{i 2}^{n+1} \widetilde{f}_{(i+1) 0}^{n+1}+\widetilde{Q}_{i 1}^{n+1} \widetilde{p}_{i 2}^{n+1} \widetilde{f}_{(i+1) 0}^{n+1} \\
= & \widetilde{Q}_{i 0}^{n+1} \widetilde{p}_{i 2}^{n+1} \widetilde{f}_{(i+1) 0}^{n+1},
\end{aligned}
$$

$$
\begin{aligned}
& \frac{d}{d \tau} \widetilde{Q}_{(i+1) 1}^{n+1}=\sum_{\kappa=2}^{2 i-2} \widetilde{Q}_{i \kappa}^{n+1} \widetilde{p}_{i 0}^{n+1}\left(\widetilde{f}_{(i+1)(\kappa-1)}^{n+1}-\widetilde{f}_{(i+1)(\kappa-2)}^{n+1}\right) \\
& +\sum_{\kappa=2}^{2 i-2}\left(\widetilde{Q}_{i(\kappa+1)}^{n+1}-\widetilde{Q}_{i \kappa}^{n+1}\right) \widetilde{p}_{i 0}^{n+1} \widetilde{f}_{(i+1)(\kappa-1)}^{n+1} \\
& +\widetilde{Q}_{i 1}^{n+1} \widetilde{p}_{i 0}^{n+1} \widetilde{f}_{(i+1) 0}^{n+1}+\left(\widetilde{Q}_{i 2}^{n+1}-\widetilde{Q}_{i 1}^{n+1}\right) \widetilde{p}_{i 0}^{n+1} \widetilde{f}_{(i+1) 0}^{n+1} \\
& +\sum_{\kappa=1}^{2 i-2} \widetilde{Q}_{i \kappa}^{n+1} \widetilde{p}_{i 1}^{n+1}\left(\widetilde{f}_{(i+1) \kappa}^{n+1}-\widetilde{f}_{(i+1)(\kappa-1)}^{n+1}\right) \\
& +\sum_{\kappa=1}^{2 i-2}\left(\widetilde{Q}_{i(\kappa+1)}^{n+1}-\widetilde{Q}_{i \kappa}^{n+1}\right) \widetilde{p}_{i 1}^{n+1} \widetilde{f}_{(i+1) \kappa}^{n+1} \\
& +\widetilde{Q}_{i 0}^{n+1} \widetilde{p}_{i 1}^{n+1} \widetilde{f}_{(i+1) 0}^{n+1}+\widetilde{Q}_{i 1}^{n+1} \widetilde{p}_{i 1}^{n+1} \widetilde{f}_{(i+1) 0}^{n+1} \\
& +\sum_{\kappa=1}^{2 i-2} \widetilde{Q}_{i \kappa}^{n+1} \widetilde{p}_{i 2}^{n+1}\left(\widetilde{f}_{(i+1)(\kappa+1)}^{n+1}-\widetilde{f}_{(i+1) \kappa}^{n+1}\right) \\
& +\sum_{\kappa=1}^{2 i-2}\left(\widetilde{Q}_{i(\kappa+1)}^{n+1}-\widetilde{Q}_{i \kappa}^{n+1}\right) \widetilde{p}_{i 2}^{n+1} \widetilde{f}_{(i+1)(\kappa+1)}^{n+1} \\
& +\widetilde{Q}_{i 0}^{n+1} \widetilde{p}_{i 2}^{n+1}\left(\widetilde{f}_{(i+1) 1}^{n+1}-\widetilde{f}_{(i+1) 0}^{n+1}\right) \\
& +\widetilde{Q}_{i 1}^{n+1} \widetilde{p}_{i 2}^{n+1} \widetilde{f}_{(i+1) 1}^{n+1}=\widetilde{Q}_{i 0}^{n+1} \widetilde{p}_{i 1}^{n+1} \widetilde{f}_{(i+1) 0}^{n+1} \\
& +\widetilde{Q}_{i 0}^{n+1} \widetilde{p}_{i 2}^{n+1}\left(\widetilde{f}_{(i+1) 1}^{n+1}-\widetilde{f}_{(i+1) 0}^{n+1}\right), \\
& \because \sum_{h=0}^{2 i} \widetilde{Q}_{(i+1) h}^{n+1}=1, \quad \therefore \sum_{h=0}^{2 i} \frac{d}{d \tau} \widetilde{Q}_{(i+1) h}^{n+1}=0 .
\end{aligned}
$$

By (17), (18), and (19), it is noted that

$$
\begin{aligned}
\frac{d}{d \tau} \widetilde{Q}_{(i+1) 0}^{n+1} & =-\frac{d}{d \tau} \widetilde{Q}_{(i+1) 1}^{n+1}-\frac{d}{d \tau} \widetilde{Q}_{(i+1) 2}^{n+1} \\
& =-\widetilde{Q}_{i 0}^{n+1} \widetilde{p}_{i 1}^{n+1} \widetilde{f}_{(i+1) 0}^{n+1}-\widetilde{Q}_{i 0}^{n+1} \widetilde{p}_{i 2}^{n+1} \widetilde{f}_{(i+1) 1}^{n+1}
\end{aligned}
$$

Using (17), (18), and (19), the first-order and second-order derivatives of $E\left(\widetilde{X}_{i}^{n+1}\right)$ are given by

$$
\begin{aligned}
\frac{d}{d \tau} E\left(\widetilde{X}_{i+1}^{n+1}\right) & =\sum_{h=1}^{2 i} h \frac{d}{d \tau} \widetilde{Q}_{(i+1) h}^{n+1} \\
& =\frac{d}{d \tau} \widetilde{Q}_{(i+1) 1}^{n+1}+2 \frac{d}{d \tau} \widetilde{Q}_{(i+1) 2}^{n+1} \\
& =\widetilde{Q}_{i 0}^{n+1} \widetilde{p}_{i 1}^{n+1} \widetilde{f}_{(i+1) 0}^{n+1}+\widetilde{Q}_{i 0}^{n+1} \widetilde{p}_{i 2}^{n+1}\left(\widetilde{f}_{(i+1) 1}^{n+1}+\widetilde{f}_{(i+1) 0}^{n+1}\right) \\
& >0
\end{aligned}
$$




$$
\begin{aligned}
\frac{d^{2}}{d \tau^{2}} E\left(\widetilde{X}_{i+1}^{n+1}\right)= & \widetilde{Q}_{i 0}^{n+1}\left(\widetilde{p}_{i 1}^{n+1}-\widetilde{p}_{i 2}^{n+1}\right) \widetilde{f}_{(i+1) 0}^{n+1}+\widetilde{Q}_{i 1}^{n+1} \widetilde{p}_{i 1}^{n+1} \widetilde{f}_{(i+1) 0}^{n+1} \\
& +\widetilde{Q}_{i 1}^{n+1} \widetilde{p}_{i 2}^{n+1} \widetilde{f}_{(i+1) 1}^{n+1}+\widetilde{Q}_{i 0}^{n+1} \widetilde{p}_{i 2}^{n+1} \widetilde{f}_{(i+1) 1}^{n+1} \\
& +\left(\widetilde{Q}_{i 0}^{n+1}+\widetilde{Q}_{i 1}^{n+1}\right) \widetilde{p}_{i 2}^{n+1} \widetilde{f}_{(i+1) 0}^{n+1} \\
> & 0 .
\end{aligned}
$$

Again, from formula (4), the result is established by

$$
\frac{d}{d \tau} E\left(\widetilde{W}_{i}^{n+1}\right)>0, \quad \frac{d^{2}}{d \tau^{2}} E\left(\widetilde{W}_{i}^{n+1}\right)>0 .
$$

(3) The waiting time at time $S_{\zeta}^{n}, \zeta>i\left(\pi_{\zeta+1}^{n+1}\right)$, is monotonically increasing convex function of $\tau$.

First, the following two equations are established by induction:

$$
\begin{aligned}
& \sum_{h=\kappa}^{2 v} \frac{d}{d \tau} \widetilde{Q}_{(i+v) h}^{n+1}>0, \quad 1 \leq \kappa \leq 2 v, v \geq 2, \\
& \sum_{h=\kappa}^{2 v} \frac{d^{2}}{d \tau^{2}} \widetilde{Q}_{(i+v) h}^{n+1}>0, \quad 1 \leq \kappa \leq 2 v, \quad v \geq 2 .
\end{aligned}
$$

For the base case, when $v=2$, applying (3), (17), (18), and (19), it is found that

$$
\begin{aligned}
\frac{d}{d \tau} \widetilde{Q}_{(i+2) 4}^{n+1}= & \widetilde{p}_{(i+1) 2}^{n+1} \widetilde{f}_{(i+2) 0}^{n+1} \frac{d}{d \tau} \widetilde{Q}_{(i+1) 2}^{n+1} \\
= & \widetilde{Q}_{i 0}^{n+1} \widetilde{p}_{i 2}^{n+1} \widetilde{f}_{(i+1) 0}^{n+1} \widetilde{p}_{(i+1) 2}^{n+1} \widetilde{f}_{(i+2) 0}^{n+1}>0 \\
\frac{d}{d \tau} \widetilde{Q}_{(i+2) 3}^{n+1}= & \widetilde{p}_{(i+1) 1}^{n+1} \widetilde{f}_{(i+2) 0}^{n+1} \frac{d}{d \tau} \widetilde{Q}_{(i+1) 2}^{n+1} \\
& +\widetilde{p}_{(i+1) 2}^{n+1} \widetilde{f}_{(i+2) 1}^{n+1} \frac{d}{d \tau} \widetilde{Q}_{(i+1) 2}^{n+1} \\
& +\widetilde{p}_{(i+1) 2}^{n+1} \widetilde{f}_{(i+2) 0}^{n+1} \frac{d}{d \tau} \widetilde{Q}_{(i+1) 1}^{n+1} \\
= & \widetilde{Q}_{i 0}^{n+1} \widetilde{p}_{i 2}^{n+1} \widetilde{f}_{(i+1) 0}^{n+1}\left(\widetilde{p}_{(i+1) 1}^{n+1}-\widetilde{p}_{(i+1) 2}^{n+1}\right) \widetilde{f}_{(i+2) 0}^{n+1} \\
& +\widetilde{Q}_{i 0}^{n+1} \widetilde{p}_{i 2}^{n+1} \widetilde{f}_{(i+1) 0}^{n+1} \widetilde{p}_{(i+1) 2}^{n+1} \widetilde{f}_{(i+2) 1}^{n+1} \\
& +\widetilde{Q}_{i 0}^{n+1} \widetilde{p}_{i 1}^{n+1} \widetilde{f}_{(i+1) 0}^{n+1} \widetilde{p}_{(i+1) 2}^{n+1} \widetilde{f}_{(i+2) 0}^{n+1} \\
& +\widetilde{Q}_{i 0}^{n+1} \widetilde{p}_{i 2}^{n+1} \widetilde{f}_{(i+1) 1}^{n+1} \widetilde{p}_{(i+1) 2}^{n+1} \widetilde{f}_{(i+2) 0}^{n+1} .
\end{aligned}
$$

Thus, $(d / d \tau) \widetilde{Q}_{(i+2) 3}^{n+1}+(d / d \tau) \widetilde{Q}_{(i+2) 4}^{n+1}>0$.

Combining equations (13) and (25),

$\frac{d^{2}}{d \tau^{2}} \widetilde{Q}_{(i+2) 4}^{n+1}=\left(\widetilde{Q}_{i 0}^{n+1}+\widetilde{Q}_{i 1}^{n+1}\right) \widetilde{p}_{i 2}^{n+1} \widetilde{f}_{(i+1) 0}^{n+1} \widetilde{p}_{(i+1) 2}^{n+1} \widetilde{f}_{(i+2) 0}^{n+1}>0$.
According to the assumption, $\widetilde{p}_{i 1}^{n+1} \geq \widetilde{p}_{i 2}^{n+1}$; hence,

$$
\begin{aligned}
& \frac{d^{2}}{d \tau^{2}} \widetilde{Q}_{(i+2) 3}^{n+1}+\frac{d^{2}}{d \tau^{2}} \widetilde{Q}_{(i+2) 4}^{n+1} \\
&=\left(\widetilde{Q}_{i 0}^{n+1}+\widetilde{Q}_{i 1}^{n+1}\right) \widetilde{p}_{i 2}^{n+1} \widetilde{f}_{(i+1) 0}^{n+1} \widetilde{p}_{(i+1) 1}^{n+1} \widetilde{f}_{(i+2) 0}^{n+1} \\
& \quad+\left(\widetilde{Q}_{i 0}^{n+1}+\widetilde{Q}_{i 1}^{n+1}\right) \widetilde{p}_{i 2}^{n+1} \widetilde{f}_{(i+1) 0}^{n+1} \widetilde{p}_{(i+1) 2}^{n+1} \widetilde{f}_{(i+2) 1}^{n+1} \\
& \quad+\left(\widetilde{Q}_{i 0}^{n+1}+\widetilde{Q}_{i 1}^{n+1}\right) \widetilde{p}_{i 1}^{n+1} \widetilde{f}_{(i+1) 0}^{n+1} \widetilde{p}_{(i+1) 2}^{n+1} \widetilde{f}_{(i+2) 0}^{n+1} \\
& \quad+\left(\widetilde{Q}_{i 0}^{n+1}+\widetilde{Q}_{i 1}^{n+1}\right) \widetilde{p}_{i 2}^{n+1} \widetilde{f}_{(i+1) 1}^{n+1} \widetilde{p}_{(i+1) 2}^{n+1} \widetilde{f}_{(i+2) 0}^{n+1} \\
&-\widetilde{Q}_{i 0}^{n+1} \widetilde{p}_{i 2}^{n+1} \widetilde{f}_{(i+1) 0}^{n+1} \widetilde{p}_{(i+1) 2}^{n+1} \widetilde{f}_{(i+2) 0}^{n+1}>0 .
\end{aligned}
$$

In the same way, $\sum_{h=\kappa}^{4}(d / d \tau) \widetilde{\mathrm{Q}}_{(i+2) h}^{n+1}>0, \kappa=1,2$;

$$
\sum_{h=\kappa}^{4} \frac{d^{2}}{d \tau^{2}} \widetilde{\mathrm{Q}}_{(i+2) h}^{n+1}>0, \quad \kappa=1,2 .
$$

Due to the limited space and tedious computation process, the concrete steps are omitted.

Suppose that (24) holds for $v<\alpha(\alpha>2)$; the next step is the proof that they also hold for $v=\alpha$.

Consider $\kappa=2 \alpha$; from equations (3) and (17) and the hypothesis, it is easy to get

$$
\begin{gathered}
\frac{d}{d \tau} \widetilde{Q}_{(i+\alpha) 2 \alpha}^{n+1}=\widetilde{p}_{(i+\alpha-1) 2}^{n+1} \widetilde{f}_{(i+\alpha) 0}^{n+1} \frac{d}{d \tau} \widetilde{Q}_{(i+\alpha-1)(2 \alpha-2)}^{n+1}>0, \\
\frac{d^{2}}{d \tau^{2}} \widetilde{Q}_{(i+\alpha) 2 \alpha}^{n+1}=\widetilde{p}_{(i+\alpha-1) 2}^{n+1} \widetilde{f}_{(i+\alpha) 0}^{n+1} \frac{d^{2}}{d \tau^{2}} \widetilde{Q}_{(i+\alpha-1)(2 \alpha-2)}^{n+1}>0 .
\end{gathered}
$$

When $2 \leq \kappa<2 \alpha$, from $\sum_{h=0}^{2 i+2 \alpha-4} \widetilde{\mathrm{Q}}_{(i+\alpha-1) h}^{n+1}=1$ and (17), it can be obtained that $\sum_{h=0}^{2 \alpha-2}(d / d \tau) \widetilde{Q}_{(i+\alpha-1) h}^{n+1}=0$. Using (3) and (17) and the hypothesis, one can get

$$
\begin{aligned}
\sum_{h=\kappa}^{2 \alpha} \frac{d}{d \tau} \widetilde{Q}_{(i+\alpha) h}^{n+1}= & \sum_{h=\kappa}^{2 \alpha} \sum_{l=h}^{2 \alpha-2} \frac{d}{d \tau} \widetilde{Q}_{(i+\alpha-1) l}^{n+1} \widetilde{p}_{(i+\alpha-1) 0}^{n+1} \widetilde{f}_{(i+\alpha)(l-h)}^{n+1} \\
& +\sum_{h=\kappa l=h-1}^{2 \alpha} \sum^{2 \alpha-2} \frac{d}{d \tau} \widetilde{Q}_{(i+\alpha-1) l}^{n+1} \widetilde{p}_{(i+\alpha-1) 1}^{n+1} \widetilde{f}_{(i+\alpha)(l-h+1)}^{n+1} \\
& +\sum_{h=\kappa l=h-2}^{2 \alpha} \sum^{2 \alpha-2} \frac{d}{d \tau} \widetilde{Q}_{(i+\alpha-1) l}^{n+1} \widetilde{p}_{(i+\alpha-1) 2}^{n+1} \widetilde{f}_{(i+\alpha)(l-h+2)}^{n+1} \\
= & \widetilde{p}_{(i+\alpha-1) 0}^{n+1} \sum_{l=0}^{2 \alpha-\kappa-2} \widetilde{f}_{(i+\alpha) l}^{n+1} \sum_{h=\kappa+l}^{2 \alpha-2} \frac{d}{d \tau} \widetilde{Q}_{(i+\alpha-1) h}^{n+1} \\
& +\widetilde{p}_{(i+\alpha-1) 1}^{n+1} \sum_{l=0}^{2 \alpha-\kappa-1} \widetilde{f}_{(i+\alpha) l}^{n+1} \sum_{h=\kappa+l-1}^{2 \alpha-2} \frac{d}{d \tau} \widetilde{Q}_{(i+\alpha-1) h}^{n+1} \\
& +\widetilde{p}_{(i+\alpha-1) 2}^{n+1} \sum_{l=0}^{2 \alpha-\kappa} \widetilde{f}_{(i+\alpha) l}^{n+1} \sum_{h=\kappa+l-2}^{2 \alpha-2} \frac{d}{d \tau} \widetilde{Q}_{(i+\alpha-1) h}^{n+1}>0 .
\end{aligned}
$$

Differentiate with respect to $\tau$; then $\sum_{h=\kappa}^{2 \alpha}\left(d^{2} / d \tau^{2}\right) \widetilde{Q}_{(i+\alpha) h}^{n+1}>0$. 
As for $\kappa=1$, similar to the previous discussion,

$$
\begin{aligned}
& \sum_{h=1}^{2 \alpha} \frac{d}{d \tau} \widetilde{Q}_{(i+\alpha) h}^{n+1}=\frac{d}{d \tau} \widetilde{Q}_{(i+\alpha) 1}^{n+1}+\sum_{h=2}^{2 \alpha} \frac{d}{d \tau} \widetilde{Q}_{(i+\alpha) h}^{n+1} \\
& =\sum_{l=1}^{2 \alpha-2} \frac{d}{d \tau} \widetilde{Q}_{(i+\alpha-1) l}^{n+1} \widetilde{p}_{(i+\alpha-1) 0}^{n+1} \widetilde{f}_{(i+\alpha)(l-1)}^{n+1} \\
& +\sum_{l=0}^{2 \alpha-2} \frac{d}{d \tau} \widetilde{Q}_{(i+\alpha-1) l}^{n+1} \widetilde{p}_{(i+\alpha-1) 1}^{n+1} \widetilde{f}_{(i+\alpha) l}^{n+1} \\
& +\sum_{l=0}^{2 \alpha-2} \frac{d}{d \tau} \widetilde{Q}_{(i+\alpha-1) l}^{n+1} \widetilde{p}_{(i+\alpha-1) 2}^{n+1} \widetilde{f}_{(i+\alpha)(l+1)}^{n+1} \\
& +\widetilde{p}_{(i+\alpha-1) 0}^{n+1} \sum_{l=0}^{2 \alpha-4} \widetilde{f}_{(i+\alpha) l}^{n+1} \sum_{h=l+2}^{2 \alpha-2} \frac{d}{d \tau} \widetilde{Q}_{(i+\alpha-1) h}^{n+1} \\
& +\widetilde{P}_{(i+\alpha-1) 1}^{n+1} \sum_{l=0}^{2 \alpha-3} \widetilde{f}_{(i+\alpha) l}^{n+1} \sum_{h=l+1}^{2 \alpha-2} \frac{d}{d \tau} \widetilde{Q}_{(i+\alpha-1) h}^{n+1} \\
& +\widetilde{p}_{(i+\alpha-1) 2}^{n+1} \sum_{l=1}^{2 \alpha-2} \widetilde{f}_{(i+\alpha) l}^{n+1} \sum_{h=l}^{2 \alpha-2} \frac{d}{d \tau} \widetilde{Q}_{(i+\alpha-1) h}^{n+1} \\
& =\widetilde{p}_{(i+\alpha-1) 0}^{n+1} \sum_{l=0}^{2 \alpha-3} \widetilde{f}_{(i+\alpha) l}^{n+1} \sum_{h=l+1}^{2 \alpha-2} \frac{d}{d \tau} \widetilde{Q}_{(i+\alpha-1) h}^{n+1} \\
& +\widetilde{p}_{(i+\alpha-1) 1}^{n+1} \sum_{l=1}^{2 \alpha-2} \widetilde{f}_{(i+\alpha) l}^{n+1} \sum_{h=l}^{2 \alpha-2} \frac{d}{d \tau} \widetilde{Q}_{(i+\alpha-1) h}^{n+1} \\
& +\widetilde{p}_{(i+\alpha-1) 2}^{n+1} \sum_{l=2}^{2 \alpha-1} \widetilde{f}_{(i+\alpha) l}^{n+1} \sum_{h=l-1}^{2 \alpha-2} \frac{d}{d \tau} \widetilde{Q}_{(i+\alpha-1) h}^{n+1}>0 .
\end{aligned}
$$

Differentiate on both sides; $\sum_{h=1}^{2 \alpha}\left(d^{2} / d \tau^{2}\right) \widetilde{Q}_{(i+\alpha) h}^{n+1}>0$.

Equation (24) hold from induction.

Calculating the first and second derivatives of $E\left(\widetilde{X}_{i+v}^{n+1}\right)$ following equations (17) and (24),

$$
\begin{aligned}
\frac{d}{d \tau} E\left(\widetilde{X}_{i+v}^{n+1}\right) & =\sum_{h=1}^{2 i+2 v-2} h \frac{d}{d \tau} \widetilde{Q}_{(i+v) h}^{n+1} \\
& =\sum_{h=1}^{2 i+2 v-2} \sum_{l=h}^{2 i+2 v-2} \frac{d}{d \tau} \widetilde{Q}_{(i+v) l}^{n+1}>0 \\
& \frac{d^{2}}{d \tau^{2}} E\left(\widetilde{X}_{i+v}^{n+1}\right)>0 .
\end{aligned}
$$

Hence, from formula (4), the result is followed by

$$
\frac{d}{d \tau} E\left(\widetilde{W}_{i}^{n+1}\right)>0, \quad \frac{d^{2}}{d \tau^{2}} E\left(\widetilde{W}_{i}^{n+1}\right)>0
$$

(4) The overtime is a monotonically increasing convex function with respect to $\tau$.
From (3), $(d / d \tau) E\left(\widetilde{X}_{n+2}^{n+1}\right)>0,\left(d^{2} / d \tau^{2}\right) E\left(\widetilde{X}_{n+2}^{n+1}\right)>0$.

From formula (5), $(d / d \tau) E\left(\widetilde{O}^{n+1}\right)>0, \quad\left(d^{2} /\right.$ $\left.d \tau^{2}\right) E\left(\widetilde{O}^{n+1}\right)>0$.

All in all, the new generated cost function is convex with respect to $\tau$.

Based on Proposition 1 and Theorem 2, the optimal $\tau \in$ $\left[S_{i-1}^{n}, S_{i}^{n}\right]$ can be easily found by any algorithm designed for convex optimization. For schedule $S^{n}, n$ best $\tau$ s are found when the $n+1$ st booking request arrives. The optimal appointment time for this patient is the one with the lowest cost. The appointment time also maximizes the profit increase considering that the revenue per patient is fixed. The next thing to do is deciding when the scheduler should stop accepting new appointments. Theorem 3 provides a sufficient condition for rejecting new requests.

Theorem 3. If scheduling a patient who is sure to come lowers the profit, the clinic should reject all future appointments.

Proof. Define $\widehat{S}_{i}^{n}$ to denote the optimal updated schedule from $S^{n}$ after accepting a patient who is sure to show up for the appointment; $\gamma_{k}^{n}$ represents the event that $k$ patients actually arrive at time $t_{n}$.

If scheduling a patient who is sure to come lowers down the profit, which can be expressed as

$$
C\left(\widehat{S}^{n+1}\right)-C\left(S^{n}\right)>r,
$$

then

$$
\begin{aligned}
& C\left(S^{n+1} \mid \gamma_{1}^{n+1}\right)-C\left(S^{n}\right)>r, \\
& C\left(S^{n+\kappa} \mid \gamma_{0}^{n+1}, \ldots, \gamma_{0}^{n+\kappa-1}, \gamma_{1}^{n+\kappa}\right)-C\left(S^{n}\right)>r \\
& \because C\left(S^{n+1} \mid \gamma_{2}^{n+1}\right)-C\left(S^{n+1} \mid \gamma_{1}^{n+1}\right) \\
& >C\left(S^{n+1} \mid \gamma_{1}^{n+1}\right)-C\left(S^{n}\right), \\
& \therefore C\left(S^{n+1}\right)-C\left(S^{n}\right) \\
& =p_{1}\left(C\left(S^{n+1} \mid \gamma_{1}^{n+1}\right)-C\left(S^{n}\right)\right) \\
& +p_{2}\left(C\left(S^{n+1} \mid \gamma_{2}^{n+1}\right)-C\left(S^{n}\right)\right) \\
& =p_{1}\left(C\left(S^{n+1} \mid \gamma_{1}^{n+1}\right)-C\left(S^{n}\right)\right) \\
& +p_{2}\left(C\left(S^{n+1} \mid \gamma_{1}^{n+1}\right)-C\left(S^{n}\right)\right) \\
& +p_{2}\left(C\left(S^{n+1} \mid \gamma_{2}^{n+1}\right)-C\left(S^{n+1} \mid \gamma_{1}^{n+1}\right)\right) \\
& >\left(p_{1}+2 p_{2}\right)\left(C\left(S^{n+1} \mid \gamma_{1}^{n+1}\right)-C\left(S^{n}\right)\right) \\
& >\left(p_{1}+2 p_{2}\right) r=E\left(R^{n+1}\right)-E\left(R^{n}\right) \text {. }
\end{aligned}
$$


Step 1. $t_{1}=0, Q_{10}^{1}=0$, testset $=\varnothing, n=1$;

Step 2. Suppose the $n+1$ st patient is of type $j \in[1, \ldots, J]$;

If $j \in$ testset, reject the patient, go to Step 2;

Else for $\left[S_{i-1}^{n}, S_{i}^{n}\right], i \in\{2, \ldots, n+1\}$, compute

$\tau_{i-1}^{n+1}=\arg \min _{\tau \in\left[S_{i-1}^{n}, S_{i}^{n}\right]} C\left(\Pi_{i-1}^{n+1}\right), \widetilde{C}_{i-1}^{n+1}=\min _{\tau \in\left[S_{i-1}^{n}, S_{i}^{n}\right]} C\left(\Pi_{i-1}^{n+1}\right)$,

$\tilde{i}=\arg \min _{i \in\{2, \ldots, n+1\}} \widetilde{C}_{i-1}^{n+1}, t_{n+1}=\tau_{(\tilde{i}-1)}^{n+1}$;

Step 3. If $P\left(S^{n+1}\right)>P\left(S^{n}\right)$, accept the patient, $n=n+1$,

testset $=\varnothing$, go to Step 2 ;

Else reject this patient, go to Step 4;

Step 4. If testset $\neq \varnothing$, testset $=[$ testset,$j]$;

If testset $=[1, \ldots, J]$, stop;

Else go to Step 5;

Step 5. Scheduling a patient who is sure to come to

$\left[S_{i-1}^{n}, S_{i}^{n}\right], i \in\{2, \ldots, n+1\}$ respectively, compute

$\widehat{C}_{i-1}^{n+1}=\min _{\tau \in\left[S_{i-1}^{n}, S_{i}^{n}\right]} C\left(\widehat{\Pi}_{i-1}^{n+1}\right), C\left(\widehat{S}^{n+1}\right)=\min _{i \in\{2, \ldots, n+1\}} \widehat{C}_{i-1}^{n+1}$.

If $C\left(\widehat{S}^{n+1}\right)-C\left(S^{n}\right)>r$, stop;

Else testset $=[j]$, go to Step 2 .

Algorithm 1

This means that

$$
\begin{aligned}
& C\left(S^{n+1} \mid \gamma_{1}^{n+1}\right)-C\left(S^{n}\right)>r \Longrightarrow P\left(S^{n}\right)>P\left(S^{n+1}\right) \\
& \because C\left(S^{n+\kappa} \mid \gamma_{1}^{n+\kappa}\right)-C\left(S^{n+\kappa} \mid \gamma_{0}^{n+1}, \ldots, \gamma_{0}^{n+\kappa-1}, \gamma_{1}^{n+\kappa}\right) \\
& \quad>C\left(S^{n+\kappa-1}\right)-C\left(S^{n}\right), \quad \kappa \geq 2, \\
& \therefore C\left(S^{n+\kappa} \mid \gamma_{1}^{n+\kappa}\right)-C\left(S^{n+\kappa-1}\right) \\
& \quad>C\left(S^{n+\kappa} \mid \gamma_{0}^{n+1}, \ldots, \gamma_{0}^{n+\kappa-1}, \gamma_{1}^{n+\kappa}\right)-C\left(S^{n}\right)>r .
\end{aligned}
$$

It is equivalent to $P\left(S^{n+\kappa}\right)>P\left(S^{n+\kappa-1}\right)$.

The results follow from the above inequality.

Theorem 3 provides a stopping criterion for rejecting new arrivals and a sufficient condition that the objective function is unimodal.

3.2. Sequential Appointment Scheduling Algorithm. Based on Proposition 1 and Theorems 2 and 3, each call-in request is scheduled to a time to maximize the profit increase. If scheduling a patient who is sure to come or scheduling a patient of any type lowers the profit, the algorithm terminates. The concrete steps are as shown in Algorithm 1.

The proposed algorithm first initializes the input parameter, and the first patient is arranged at time 0 based on Proposition 1. Every new booking request is attempted to schedule to $\left[S_{i-1}^{n}, S_{i}^{n}\right], i \in\{2, \ldots, n+1\}$; the minimum $\widetilde{C}_{i-1}^{n+1}$ in each $\left[S_{i-1}^{n}, S_{i}^{n}\right]$ is obtained based on the convexity of the cost function. $t_{n+1}$ is the selected time with minimum cost; if $P\left(S^{n+1}\right)>P\left(S^{n}\right)$, then accept this patient and arrange him or her at $t_{n+1}$; otherwise, check whether set "testset" is empty. If testset $=\varnothing$, testify whether scheduling a patient who is sure to come lowers down the profit. The algorithm will terminate according to Theorem 3 if $C\left(\widehat{S}^{n+1}\right)-C\left(S^{n}\right)>r$ holds. Put $j$ into set "testset" until testset $=[1, \ldots, J]$, if testset $\neq \varnothing$. The algorithm stops with any further acceptances lowering the profit.

\section{Numerical Analysis}

To demonstrate the effectiveness of the proposed algorithm, a randomly generated call-in sequence is analyzed to display the trends of the objectives. The average performance of 1000 random sequences is used to compare with the commonly used heuristics in practice. The impacts of no-shows and the walk-in probability on the performance are investigated. The basic parameters for the numerical experiments are as follows: the length of the session is $T=48$ (typically, the clinic is open for 4 hours in the morning and the afternoon; the average service time is five minutes, standardized at 1), the revenue per patient is $r=1$, and the waiting $\cos t c_{w}$ and overtime cost $c_{o}$ per unit of time are 0.2 and 1.5, respectively; (an overtime cost larger than the marginal revenue per patient avoids an infinite increase in patients; otherwise, the clinic will always benefit from serving additional patients through extending the working time.) Suppose that $J=$ 4 ; then the corresponding no-show probability is $[0.5,0.4$, $0.3,0.2]$. It is assumed that each patient arrives independently, and walk-in patients are independent of patients with appointments. The basic walk-in probability is 0.25 . The minimum $\widetilde{C}_{i-1}^{n+1}$ in the interval $\left[S_{i-1}^{n}, S_{i}^{n}\right]$ is derived by the Matlab 7.8 (product of MathWorks Company, Natick, Massachusetts, USA) optimization solver "fmincon," which implements the sequential quadratic programming algorithm.

4.1. Illustrating Example. Figure 2 presents the changing trend of the objective function and the appointment time allocated to each patient for a specific call-in sequence. The abscissa represents the patient type in the call-in sequence; for example, 4, 4, 1 means that the first three patients belong to types $4,4,1$; the left ordinate represents the profit, and 


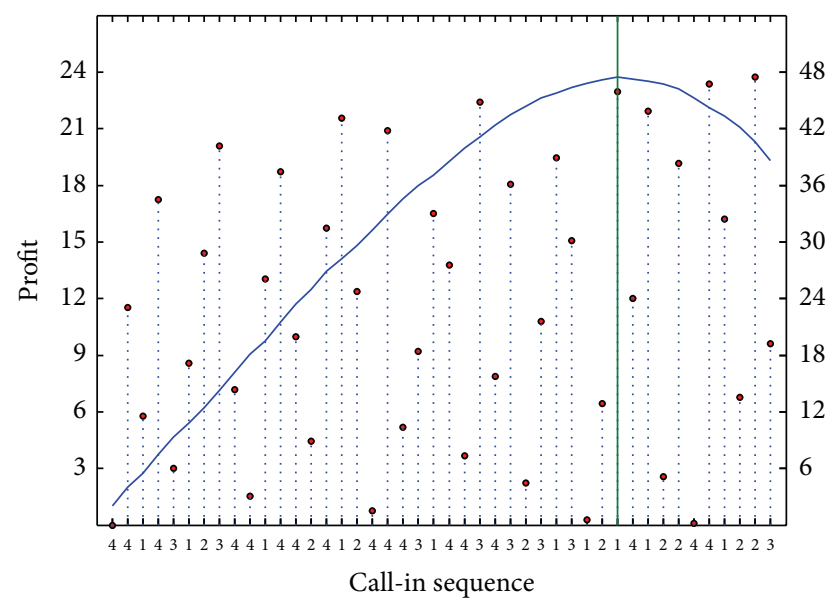

FIgURE 2: Illustration of the schedule and the expected profit evolution.

TABLE 1: Statistics of 1000 random sequences.

\begin{tabular}{lcc}
\hline & Scheduled patients & Profit \\
\hline Maximum & 37 & 23.84 \\
Minimum & 34 & 22.93 \\
\hline Average & 35.378 & 23.43 \\
\hline
\end{tabular}

the right ordinate represents the appointment time. The curve displays the profit changes during the call-in process. The dot is the appointment time provided by the algorithm for each booking request. Ten additional patients are used to display the trend of the profit after the stopping criterion is achieved. It is shown that the optimal schedule accepts 34 patients, and the profit function monotonically increases initially and decreases later. After the terminating criterion, the profit decreases quickly, and half of the 10 additional patients are arranged at the end of the clinic session. Intuitively speaking, it is of no use incorporating them into the schedule when the system is congested except increasing the waiting time of prescheduled patients. These extra patients are served in overtime when the system is overloaded. Theorem 3 provides sufficient conditions that the objective function is unimodal, but the algorithm stops if any type of patient lowers the profit according to the above procedure. 192 of 1000 random generated call-in sequences satisfy the conditions in Theorem 3, and no better solutions are observed for the 10 more patients in the other sequences. The statistics of performance of interest are presented in Table 1, and it is noted that the proposed approach is very stable with small fluctuations. The average of 100 random generated call-in sequences is adopted in a subsequent experiment.

\subsection{Comparison with Commonly Used Heuristics. Cayirli} et al. [18] sum up some appointment rules, including the IBFI (individual block/fixed interval), 2BEG (individual block, fixed interval rule with an initial block of two patients), OFFSET (individual block/variable interval rule, where initial $k_{1}-$ 1 patients are scheduled earlier, and the rest of the patients are scheduled later), DOME (individual block/variable interval rule, where initial $k_{1}-1$ patients are scheduled earlier, patients $k_{1}+1$ through $k_{2}-1$ are scheduled later, and the rest are scheduled earlier), 2BGDM (combination of the 2BEG and the DOME rules), MBFI (multiple block/fixed interval rule calls two patients at a time with the interval length equal to twice the mean service time), and MBDM (combination of the MBFI and DOME rules). These rules are listed in Table 2 to compare with the proposed policy. No patients are scheduled before the beginning of the session because unpunctual patients are not included. $k_{1}=24, k_{2}=40$, $\beta_{1}=0.1, \beta_{2}=0.2$, and $\beta_{3}=0.05$ are used for Cayirli's rule, and the other parameters are identical to the basic case. The optimal scheduled number and the corresponding profit of all the heuristics are obtained to compare with the algorithm. The numerical results show that the heuristics accept more patients, but they lower the net expected profit significantly. IBFI performs best in all the heuristics considered, but it still leads to a fall in profit by $28 \%$.

4.3. Impacts of No-Show Probability. The impact of no-shows is analyzed from two aspects: the mean and the variance. Four groups of no-show probability are used to display the results:

(1) impact of mean: $[0.65,0.55,0.45,0.35],[0.35,0.25$, $0.15,0.05]$

(2) impact of variance: $[0.4,0.367,0.333,0.3],[0.65,0.45$, $0.25,0.05]$.

Table 3 summarizes the performances for these four groups of the no-show probability. It is noted that a large noshow probability can incur more uncertainty, bringing about a decrease in profit. An interesting result which is contrary to the intuition is that when the mean of the no-show probability is fixed, a small fluctuation lowers the profit. The patients with large show-up and no-show probabilities can bring more profits because of small uncertainty. That is to say, the clinic will benefit from reducing the average no-show probability and controlling the difference between the maximum and minimum no-show probabilities.

4.4. Impacts of Weight of Patient Type. This subsection investigates the influence of the weight of patient type on the total profit. The no-show probability is the same as in the basic case. The four types of weight are analyzed as follows: (1) $4: 3: 2: 1$; (2) $1: 2: 3: 4$; (3) $1: 2: 2: 1 ;$ (4) $2: 1: 1: 2$.

Table 4 summarizes the statistics for these four groups of weight. Consistent with the previous results, (2) brings more profit than (1) by cutting down the weight given to patients with a high no-show probability. The average noshow probability of (3) and (4) is the same, but the large fluctuation produces more profits.

4.5. Impacts of Walk-In Patients. Table 5 displays the performances for six walk-in probabilities: $0,0.1,0.2,0.3,0.4$, and 0.5 . It is observed that a large walk-in probability with more uncertainty leads to a decrease in the profit. Walk-in appointments are an alternative approach to compensate for no-shows, but overbooking is more efficient. This implies that 
TABLE 2: Comparison of overbooking and walk-in policies on system performance.

\begin{tabular}{|c|c|c|c|}
\hline Appointment rule & Interval length & Scheduled patients & Profit \\
\hline The proposed algorithm & - & 35.26 & 23.45 \\
\hline IBFI & $s_{i}=(i-1) \mu^{-1}$ & 40.24 & 16.96 \\
\hline OFFSET & $\begin{array}{l}s_{1}=0 \\
s_{i}-s_{i-1}=\left(1-\beta_{1}\right) \mu^{-1}, 2 \leq i \leq k_{1} \\
s_{i}-s_{i-1}=\left(1+\beta_{2}\right) \mu^{-1}, i>k_{1}\end{array}$ & 42.89 & 16.37 \\
\hline DOME & $\begin{array}{l}s_{1}=0 \\
s_{i}-s_{i-1}=\left(1-\beta_{1}\right) \mu^{-1}, 2 \leq i \leq k_{1} \\
s_{i}-s_{i-1}=\left(1+\beta_{2}\right) \mu^{-1}, k_{1}<i \leq k_{2} \\
s_{i}-s_{i-1}=\left(1-\beta_{3}\right) \mu^{-1}, i>k_{2}\end{array}$ & 42.4 & 16.35 \\
\hline 2BEG & $\begin{array}{l}s_{1}=s_{2}=0 \\
s_{i}=(i-2) \mu^{-1}, i>2\end{array}$ & 40.89 & 16.26 \\
\hline 2BGDM & $\begin{array}{l}s_{1}=s_{2}=0 \\
s_{i}-s_{i-1}=\left(1-\beta_{1}\right) \mu^{-1}, 2<i \leq k_{1} \\
s_{i}-s_{i-1}=\left(1+\beta_{2}\right) \mu^{-1}, k_{1}<i \leq k_{2} \\
s_{i}-s_{i-1}=\left(1-\beta_{3}\right) \mu^{-1}, i>k_{2}\end{array}$ & 43.03 & 15.62 \\
\hline MBFI & $s_{i}=s_{i+1}=(i-1) \mu^{-1}, i=1,3,5, \ldots$ & 39.86 & 15.49 \\
\hline MBDM & $\begin{array}{l}s_{1}=s_{2}=0 \\
s_{i}-s_{i-1}=s_{i+1}-s_{i-1}=2\left(1-\beta_{1}\right) \mu^{-1}, 2<i \leq k_{1} \\
s_{i}-s_{i-1}=s_{i+1}-s_{i-1}=2\left(1+\beta_{2}\right) \mu^{-1}, k_{1}<i \leq k_{2} \\
t_{i}-t_{i-1}=t_{i+1}-t_{i-1}=2\left(1-\beta_{3}\right) \mu^{-1}, i>k_{2}\end{array}$ & 42.06 & 15.00 \\
\hline
\end{tabular}

TABLE 3: Impact of no-show probability on system performance.

\begin{tabular}{lcc}
\hline No-show probability & Scheduled patients & Profit \\
\hline $0.5,0.4,0.3,0.2$ & 35.26 & 23.45 \\
$0.65,0.55,0.45,0.35$ & 42.1 & 22.54 \\
$0.35,0.25,0.15,0.05$ & 32.07 & 24.21 \\
$0.4,0.367,0.333,0.3$ & 34.6 & 23.39 \\
$0.65,0.45,0.25,0.05$ & 36.13 & 23.64 \\
\hline
\end{tabular}

TABLE 4: Impact of patients' weight on system performance.

\begin{tabular}{lcc}
\hline No-show probability & Scheduled patients & Profit \\
\hline $1: 1: 1: 1$ & 35.26 & 23.45 \\
$4: 3: 2: 1$ & 37.28 & 23.07 \\
$1: 2: 3: 4$ & 34.13 & 23.79 \\
$1: 2: 2: 1$ & 35.16 & 23.42 \\
$2: 1: 1: 2$ & 35.51 & 23.45 \\
\hline
\end{tabular}

TABLE 5: Impact of walk-in probability on system performance.

\begin{tabular}{lcc}
\hline Walk-in probability & Scheduled patients & Profit \\
\hline 0 & 50.62 & 24.8 \\
0.1 & 43.27 & 23.91 \\
0.2 & 37.59 & 23.5 \\
0.3 & 34.02 & 23.43 \\
0.4 & 31.22 & 23.19 \\
0.5 & 28.03 & 23.16 \\
\hline
\end{tabular}

appointment scheduling is an effective approach to smooth the patient flow in the clinic; the administrator of the clinic should make some constraint on the proportion of walkin patients, allowing the clinic to provide diagnosis to more patients and gain more profits as well.

4.6. Impacts of Anticipating Future Arrivals. According to Theorem 2 and the experimental results about no-show probability, it is noted that the clinic can benefit from restricting the patients with large no-show probability. This motivates a heuristic that only patients with small no-show probability are accepted during the initial portion of the booking horizon and appointment requests with large no-show probability are considered only in the later portion when not enough future arrivals are anticipated. It is easy to see that the proof of Theorems 2 and 3 hold when future arrivals are considered; so the proposed algorithm can be implemented for this scenario. The numerical experiment generates a call-in sequence including 80 patients: type 4 will be accepted for the first 20 patients, types 3 and 4 will be accepted for the next 20 patients, and so on. Figure 3 displays the evolution of objective for a given call-in sequence denoted by the horizontal coordinate. 10 more patients are scheduled to illustrate the evolution of the profit after the stopping criteria are achieved. In this special case, it is seen that the optimal number of patients is 34 with the expected profit of 24.02. The statistic results of 100 randomly generated call-in sequences show that 33.7 patients are scheduled on average with the mean profit of 24.01. Compared with performances from Table 1, it is noted that rejecting some patients with high no-show probabilities will schedule fewer patients but will increase the expected revenue in the meanwhile. This means that future arrivals can be also viewed as an alternative approach to substitute for patients with high no-show probabilities. If the clinic has enough potential booking requests, patients with large no-show probabilities are not accepted. 


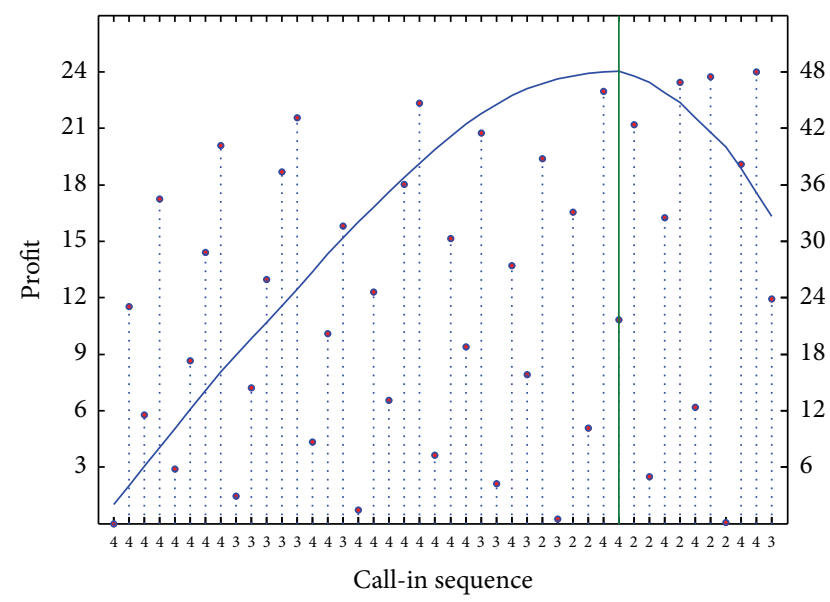

FIgURE 3: Illustration of the schedule and the expected profit anticipating future arrivals.

\section{Conclusion}

This paper develops a stochastic programming model for a sequential appointment scheduling problem with walkin patients. An algorithm terminating with the myopic optimal number of patients scheduled and the corresponding appointment time is proposed based on the convexity of cost function. A sufficient condition regarding the unimodality of the profit function is established. The numerical results show that this algorithm outperforms the commonly used heuristics in terms of the profit. Numerical results implicate that large no-show probabilities are key factors influencing the profit. Lowering down mean and variance of the no-show probabilities can bring extra profit for the clinic. Although walk-in patients can fill in gaps resulted in by no-show, overbooking is preferable in terms of total profit. Rejecting patients with large no-show probabilities when the clinic has enough potential appointments is an alternative approach to mitigate the negative effects of no-show behaviors.

Future directions can introduce other types of variability in the appointment system. An immediate direction is the inclusion of generally distributed service time to see to what extent the previous conclusion holds. Future researches should take into account other behaviors such as patients' preference, unpunctuality, and service interruption which also have significant impacts on the optimal schedule. As seen from this paper, reducing no-show behavior benefits both the clinic and patients. Intuitively speaking, the indirect waiting time between the call to make an appointment and the actual appointed day may influence the no-show probability. More accurate models should contain multiperiods to account for this effect quantitatively. It is noted that restricting walkin patients will increase the profit of the clinic, but several field studies favor the so-called open-access appointment system, which provides same-day service to the patients. This paradox stimulates further studies to decide the length of time intervals left vacant for same-day appointments and when these intervals start simultaneously.

\section{Conflict of Interests}

The authors declare that there is no conflict of interests regarding the publication of this paper.

\section{Acknowledgment}

This paper is financially supported by the National Natural Science Foundation of China (NSFC Project 71021061).

\section{References}

[1] N. T. J. Bailey, "A study of queues and appointment systems in hospital outpatient departments, with special reference to waiting times," Journal of Royal Statistics Society Series A, vol. 14, pp. 185-199, 1952.

[2] T. Cayirli and E. Veral, "Outpatient scheduling in health care: a review of literature," Production and Operations Management, vol. 12, no. 4, pp. 519-549, 2003.

[3] D. Gupta and B. Denton, "Appointment scheduling in health care: challenges and opportunities," IIE Transactions, vol. 40, no. 9, pp. 800-819, 2008.

[4] S. Kim and R. E. Giachetti, "A stochastic mathematical appointment overbooking model for healthcare providers to improve profits," IEEE Transactions on Systems, Man, and Cybernetics Part A: Systems and Humans, vol. 36, no. 6, pp. 1211-1219, 2006.

[5] X. Qu, R. L. Rardin, J. A. S. Williams, and D. R. Willis, "Matching daily healthcare provider capacity to demand in advanced access scheduling systems," European Journal of Operational Research, vol. 183, no. 2, pp. 812-826, 2007.

[6] L. LaGanga and S. R. Lawrence, "Appointment overbooking in health care clinics to improve patient service and clinic performance," Productions and Operations Management, vol. 21, no. 5, pp. 874-888, 2012.

[7] G. Kaandorp and G. Koole, "Optimal outpatient appointment scheduling," Health Care Management Science, vol. 10, no. 3, pp. 217-229, 2007.

[8] R. Hassin and S. Mendel, "Scheduling arrivals to queues: a single-server model with no-shows," Management Science, vol. 54, no. 3, pp. 565-572, 2008.

[9] T. Cayirli, K. K. Yang, and S. A. Quek, "A universal appointment rule in the presence of no-shows and walk-ins," Production and Operations Management, vol. 21, no. 4, pp. 682-697, 2012.

[10] K. Muthuraman and M. Lawley, "A stochastic overbooking model for outpatient clinical scheduling with no-shows," IIE Transactions, vol. 40, no. 9, pp. 820-837, 2008.

[11] S. Chakraborty, K. Muthuraman, and M. Lawley, "Sequential clinical scheduling with patient no-shows and general service time distributions," IIE Transactions, vol. 42, no. 5, pp. 354-366, 2010.

[12] S. Chakraborty, K. Muthuraman, and M. Lawley, "Sequential clinical scheduling with patient no-show: the impact of predefined slot structures," Socio-Economic Planning Sciences, vol. 47, no. 3, pp. 205-219, 2013.

[13] B. Zeng, A. Turkcan, J. Lin, and M. Lawley, "Clinic scheduling models with overbooking for patients with heterogeneous noshow probabilities," Annals of Operations Research, vol. 178, no. 1, pp. 121-144, 2010.

[14] A. Turkcan, B. Zeng, K. Muthuraman, and M. Lawley, "Sequential clinical scheduling with service criteria," European Journal of Operational Research, vol. 214, no. 3, pp. 780-795, 2011. 
[15] R. Kopach, P.-C. DeLaurentis, M. Lawley et al., "Effects of clinical characteristics on successful open access scheduling," Health Care Management Science, vol. 10, no. 2, pp. 111-124, 2007.

[16] D. Gupta and L. Wang, "Revenue management for a primarycare clinic in the presence of patient choice," Operations Research, vol. 56, no. 3, pp. 576-592, 2008.

[17] S. A. Erdogan and B. Denton, "Dynamic appointment scheduling of a stochastic server with uncertain demand," INFORMS Journal on Computing, vol. 25, no. 1, pp. 116-132, 2013.

[18] T. Cayirli, E. Veral, and H. Rosen, "Designing appointment scheduling systems for ambulatory care services," Health Care Management Science, vol. 9, no. 1, pp. 47-58, 2006. 


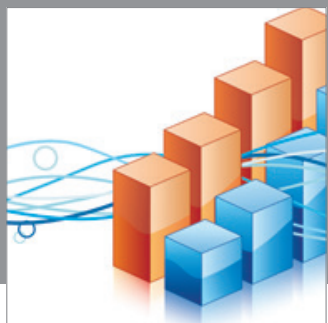

Advances in

Operations Research

mansans

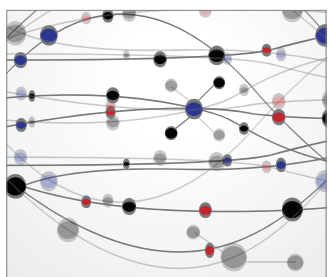

The Scientific World Journal
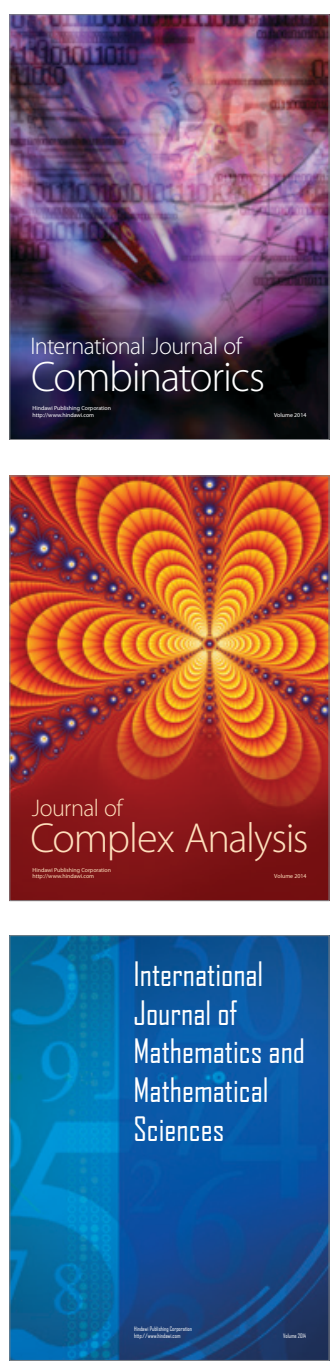
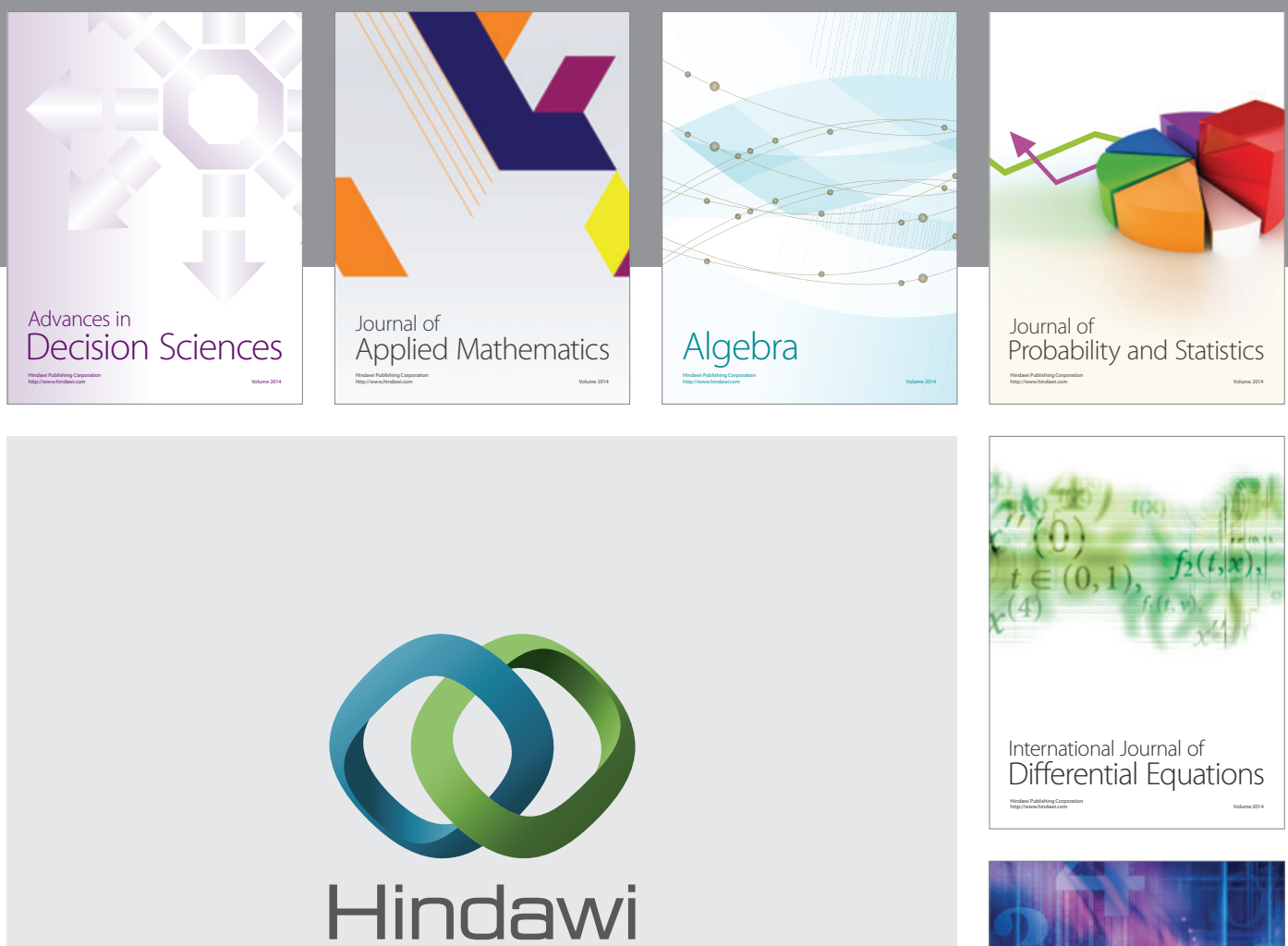

Submit your manuscripts at http://www.hindawi.com
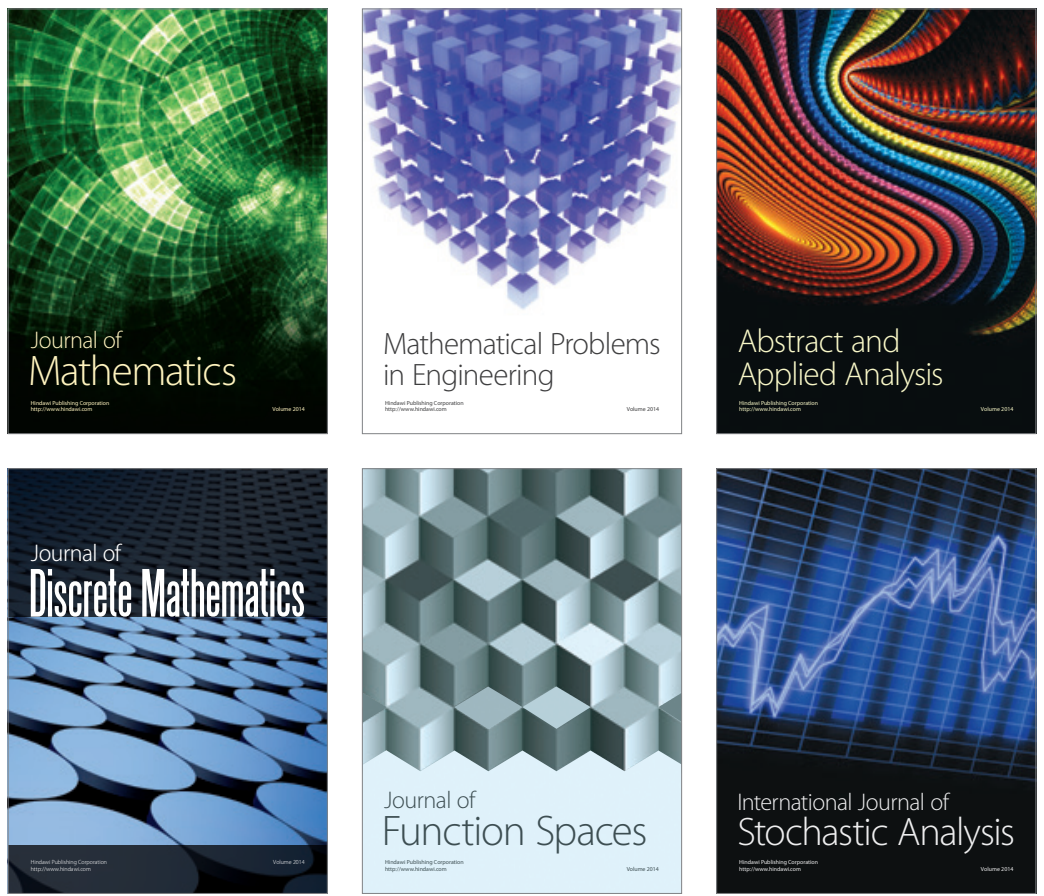

Journal of

Function Spaces

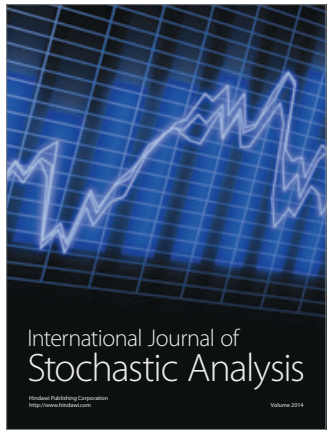

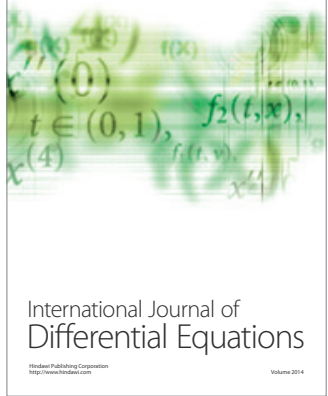
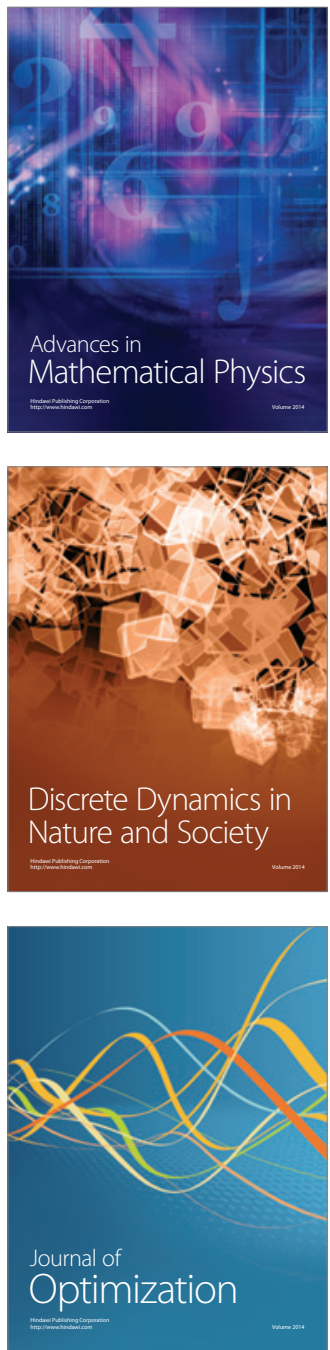